\title{
Du nakamal à la maison-cuisine : chronique socio- architecturale de Mere Lava (Vanuatu)
}

From nakamal to kitchen houses: a socio-architectural chronicle of Mere Lava

Island (Vanuatu)

\section{Marie Durand}

\section{(2) OpenEdition}

Journals

\section{Édition électronique}

URL : http://journals.openedition.org/jso/7559

DOI : $10.4000 /$ jso.7559

ISSN : $1760-7256$

Éditeur

Société des océanistes

Édition imprimée

Date de publication : 31 décembre 2016

Pagination : 223-239

ISSN : 0300-953x

Référence électronique

Marie Durand, «Du nakamal à la maison-cuisine : chronique socio-architecturale de Mere Lava

(Vanuatu) ", Journal de la Société des Océanistes [En ligne], 142-143 | 2016, mis en ligne le 31 décembre 2018, consulté le 23 avril 2019. URL : http://journals.openedition.org/jso/7559; DOI : 10.4000/ jso.7559 


\section{Du nakamal à la maison-cuisine, chronique socio- architecturale de Mere Lava (Vanuatu)}

par

Marie DURAND*

\section{RÉSUMÉ}

Dans les îles Banks, au Vanuatu, certaines des maisons des hommes ont aujourd'hui disparu. En revanche, d'autres constructions telles que les maisons-cuisine conservent une importance sociale prépondérante dans la vie quotidienne et cérémonielle. Comment comprendre ces changements architecturaux? Des maisons des hommes de la fin du XIX siècle aux maisons-cuisine contemporaines, cet article propose une analyse basée sur les données ethnographiques recueilles sur le terrain, des documents d'archives missionnaires et coloniales et l'histoire orale de l'île de Mere Lava afin de dessiner une chronique des changements sociaux et architecturaux dans cette région du Pacifique.

Mots-Clés : Vanuatu, Mere Lava, Banks, architecture, changements sociaux, maisons des hommes, maisons-cuisine

\begin{abstract}
In the Banks Islands, Vanuatu, some of the men's houses have disappeared. By contrast, some other buildings such as the kitchen houses are imbued with a primary social importance in daily and ceremonial life. How can we understand those architectural changes? Fom the end of the $19^{\text {th }}$ century's men's houses to the current kitchen houses, I propose an analysis based on ethnographical fieldwork, missionary and colonial archives and the oral history of the island of Mere Lava in order to draw a chronicle of social and architectural change in this region of the Pacific.
\end{abstract}

KeYwords: Vanuatu, Mere Lava, Banks Islands, architecture, social change, men's houses, kitchen houses
Depuis Lewis Henri Morgan (1881), de nombreux travaux ont exploré la dynamique réciproque des relations entre les hommes et leurs maisons (Bourdieu, 1972 ; Rapoport, 1976 ; Lévi-Strauss, 1987; Carsten et Hugh-Jones, 1995 ; Oliver, 2006 ; Buchli, 2013). Parmi ceuxci, certains ont interrogé plus spécifiquement les interactions entre les aspects matériels de l'architecture et la construction des personnes et des groupes sociaux dans le Pacifique (Waterson, 1990 ; Fox, 1993 ; Rodman et Rensel, 1997 ; Stasch, 2011). Si les pouvoirs étatiques coloniaux et postcoloniaux se représentent et, de manière générale, expriment leur dynamisme à travers l'architecture et la transformation des espaces et des bâtiments publics, les espaces domestiques sont aussi des lieux privilégiés dans et par lesquels les changements sociaux, historiques, économiques ou politiques sont matérialisés, confrontés et intégrés (voir par exemple Kembol et al., 1976 ; Rutz, 1984 ; Vea, 1985). Comme l'affirment avec justesse Rodman et Rensel :

"Houses can be seen as a nexus where sociocultural, economic and political forces interact, transcending disciplinary boundaries. Ordinary housing provides a focal

\footnotetext{
* Postdoctorante de la Fondation Fyssen, Centre de recherche et de documentation sur l'Océanie (AMU, CNRS, EHESS - CREDO, UMR 7308), Marseille, marie.durand@univ-amu.fr
} 
point for discovery and exploration of the stories embodied therein.» (1997: 9)

Ces histoires sont complexes et se développent à des niveaux multiples, associant réactions particulières et contextes socio-économiques plus larges. Au Vanuatu, tandis que certaines études portant sur l'architecture se sont concentrées sur la documentation des techniques de construction dites " traditionnelles", dans le but explicite de préserver ces connaissances au bénéfice des générations futures (Coiffier, 1988 : ix), d'autres ont pris en compte ses transformations afin de mettre en lumière l'aspect dynamique et "vivant » des constructions (Rodman, 1985, 1987). Elles ont montré que les maisons contribuent à créer et à matérialiser dans l'espace les relations à l'histoire et au temps des individus et des groupes (Taylor, 2008). Malgré leur importance, ces recherches se placent néanmoins à l'intérieur de limites respectivement formelles ou temporelles définies : soit parce que les bâtiments construits à partir de matériaux importés sont exclus de l'analyse, soit que l'étude se concentre temporellement sur quelques décennies tout au plus. Je suggère que le développement sur le temps long d'une étude des changements architecturaux permet de mieux percevoir la pluralité et l'imbrication des facteurs du changement ainsi que d'identifier les acteurs de sa médiation à différents niveaux (voir aussi Peltier, 2008). Cet article se penche donc, à la manière d'une chronique et à partir de l'analyse d'archives écrites, de l'histoire orale et d'une étude ethnographique, sur les changements de l'organisation spatiale et de l'architecture au Vanuatu, ce à partir des transformations qui ont pu être retracées dans l'archipel des îles Banks (province de Torba), et plus précisément à partir du cas de l'île de Mere Lava, située au sud-est des Banks.

Aujourd'hui, les maisons qui sont désignées dans cette île comme les bâtiments centraux des hameaux sont les maisons-cuisine, n-ean- kuk (figure I). La maîtrise technique associée à leur construction ainsi que leurs caractéristiques formelles s'intègrent à l'ensemble des connaissances et savoir-faire locaux définis comme coutumiers, kastom, par les habitants de l'île. Leur construction donne lieu au rassemblement ponctuel de l'ensemble des parents du commanditaire et à un certain nombre de gestes coutumiers qui les ancrent, ainsi que les personnes qui y sont associées, dans le territoire. Ces maisons-cuisine sont réalisées avec un soin particulier expliqué comme étant la garantie de leur durée dans le temps (15 à 25 ans selon les interlocuteurs interrogés) et de leur résistance aux aléas climatiques tels que les cyclones, fréquents dans la région. L'île de Mere Lava se distingue donc par rapport à nombre d'autres îles de l'archipel du Vanuatu, où les espaces définis comme cuisines peuvent être de simples abris ou des maisons érigées plus rapidement (par exemple Taylor, 2008 : 145). Enfin, l'analyse des discours contemporains produits autour de ces maisons a montré leur association avec la conception locale de la notion d'histoire, histri en bislama, la langue véhiculaire du Vanuatu (Durand, 2016). Dans ce cadre, les maisons-cuisine, à travers leur construction et leur usage, permettent l'incorporation par l'action de l'expérience historique et des réalités sociales contemporaines. Elles sont des éléments constitutifs et intrinsèques de la notion d'histri et constituent finalement de véritables potentiels d'actions futures, sous forme matérielle, pour ceux qui les construisent et les reconstruisent.

Pourtant, comme leur nom vernaculaire, $n$-ean$k u k$, inspiré de l'anglais cook (par le biais du bislama kuk) l'indique, les maisons-cuisine telles qu'elles sont aujourd'hui bâties et investies de sens sont des créations relativement récentes. Je me propose dans cet article d'éclairer la place singulière et la chronologie du développement de ces constructions à la lumière du contexte colonial et postcolonial spécifique de cette île.

Peuplée par des populations Lapita vers 1000 av. J.C., les îles Banks sont intégrées à des réseaux d'échanges inter-îles dès ces périodes anciennes (Kirch et Yen, 1982 : 260-261 ; Best, 1984 : 494) ${ }^{1}$. Ces échanges se transforment cependant à partir du milieu du XIX ${ }^{e}$ siècle, lorsque les contacts des insulaires s'intensifient avec ces nouveaux venus que sont les marchands, les recruteurs de maind'œuvre et, à partir de 1856, les missionnaires anglicans de divers origines. Les réseaux existants qui permettaient la circulation des objets, des techniques, des éléments de rituels et des personnes entre les îles sont alors durablement modifiés et intègrent localement les éléments nouveaux apportés par ces étrangers (Huffman, 1996 ; Armstrong, 1900 ; Hilliard, 1978 ; Shineberg, 1967. 1999 ; voir aussi les journaux des missionnaires Palmer, 1866 et Codrington, 1870, 1872). Les systèmes d'acquisition de statut et d'autorité ${ }^{2}$ de la région ainsi que les caractéristiques de l'organisation spatiale et de l'architecture qui leur sont associés changent alors progressivement. C'est à

1. Selon ces auteurs, on retrouve par exemple de l'obsidienne des îles Banks dans des contextes Lapita et ensuite après 1200 à Tikopia. De même à Fidji, où elle est présente après 1000 de notre ère.

2. Ces systèmes statutaires du centre-nord du Vanuatu ont été décrits comme "sociétés de grades " dans la littérature portant sur les organisations politiques coutumières au Vanuatu (Allen, 1981 ; Vienne, 1984). Dans le cas des îles Banks, je suivrai quant à moi l'exemple de Lissant Bolton qui note pour les systèmes statutaires de l'île d'Ambae l'insuffisance du terme de société de grade pour désigner l'ensemble des institutions complexes qui forment le système statutaire et dont certaines ne fonctionnent pas en terme de grades. Elle emploie ainsi le terme « status alteration system » que je choisi de traduire simplement par système statutaire (Bolton, 2003 : XXXIII). 


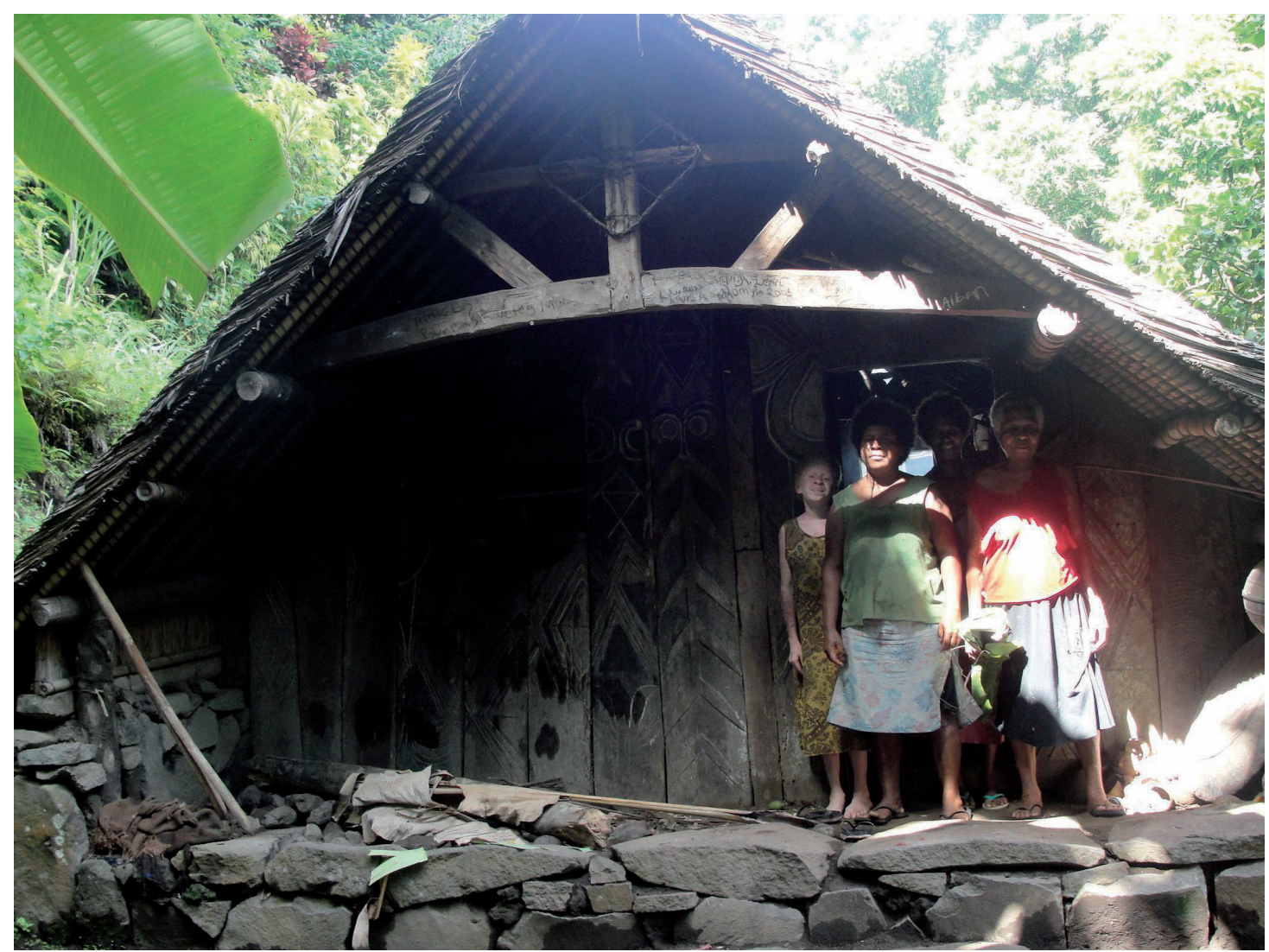

FIgURE 1. - Luisa Rosep et sa famille à la porte de leur maison-cuisine, Leveré, village de Leqel, Mere Lava (C) Marie Durand, 2011)

partir de ce moment de présence missionnaire, dans la deuxième moitié du XIX ${ }^{\mathrm{e}}$ siècle, que nous explorerons les logiques de transformation sociales et architecturales dans les îles Banks et en particulier sur l'île de Mere Lava.

\section{Maisons des hommes, système d'altération statutaire et missionnaires (deuxième moitié du XIX ${ }^{\mathrm{e}}$ siècle - début du XX $\mathrm{X}^{\mathrm{e}}$ siècle)}

\section{Les systèmes statutaires anciens et leur ancrage spatial dans les îles Banks}

Il est toujours vain de tenter de retrouver avec exactitude les traits d'une société pré-contact, tant l'imbrication des influences mutuelles, la variété et la rapidité des stratégies et des réponses individuelles ou collectives au changement rendent toute reconstruction complexe. C'est donc une description, sans aucun doute beaucoup trop homogène, de certains traits de la société du XIX ${ }^{\mathrm{e}}$ siècle en situation de contact qui sera esquissée ici, à partir d'éléments issus de l'histoire orale, cles récits et des archives missionnaires. Elle permettra néanmoins de dessiner les contours de certains changements architecturaux qui transformeront ensuite l'organisation spatiale de la région. Dans les îles Banks, comme généralement dans le nord du Vanuatu, hommes et femmes convertissaient certains biens de valeur en prestige et en autorité à travers un système complexe et compétitif d'acquisition de statut (Codrington, 1891 ; Rivers, 1914; Speiser, 1923; Deacon, 1934; Layard, 1942 ; Vienne, 1971, 1972, 1982, 1984, 1996 ; Bonnemaison, 1996 ; Bolton, 2003). Pour un homme, acquérir un statut social élevé signifiait gravir progressivement une hiérarchie de rangs ou de grades associés à des titres et à des fonctions pré-déterminées. D’autre part, les hommes intégraient aussi une ou plusieurs sociétés "secrètes " associées aux esprits ancestraux, appelés tamate en mota $^{3}$, dont la présence était matérialisée par les masques lors des danses cérémonielles (Vienne, 1971 : 319-327). Prises de grades et initiations dans les sociétés " secrètes " donnaient lieu à des cérémonies publiques appelées kolekole (Mo), qui venaient légitimer l'acquisition du nouveau sta-

3. La langue mota étant la langue de référence utilisée par la mission anglicane comme 'lingua franca' à partir de 1849 ainsi que celle utilisée dans les premiers comptes rendus ethnographiques, j'ai gardé les termes en Mota pour la description générique des grands traits communs des systèmes statutaires des îles Banks. Les termes en Mota seront ici indiqués par l'abréviation (Mo) tandis que les termes en mwerlap, langue l'île de Mere Lava, seront indiquées par l'abréviation (Mwe). 
tut tout en inscrivant l'autorité du nouveau gradé dans les lieux même de son exercice. Des plateformes de pierres ainsi que divers monuments de grade pouvaient ainsi marquer la place de danse où avait lieu la cérémonie kolekole (Mo). Le pouvoir acquis par les hommes de haut rang, conceptualisé en terme de proximité avec le monde surnaturel et exprimé par la notion de mana $(\mathrm{Mo})^{4}$, était ainsi transformé en autorité par le biais de ce que l'anthropologue Bernard Vienne nomme une " économie politique ", centrée sur la manipulation et la circulation de trois éléments essentiels : la nourriture, la monnaie de coquillage et les cochons à dents recourbées (Vienne, 1971 : 29). Les liens entre la hiérarchie des grades, nommée suqe (Mo) et les sociétés "secrètes " sont peu documentés même si l'on sait qu'il fallait être membre de certaines de ces sociétés pour pouvoir acquérir certains grades (Durrad, 1920 ; Vienne, 1971, 1972, 1982, 1984, 1996 ; Kolshus, 1999 ; Lanouguère-Bruneau 2002).

Les femmes obtenaient elles aussi statut et autorité au sein d'un système spécifique et complémentaire du système masculin (Rivers, 1914 : 130-134 ; Lanouguère-Bruneau, 2002 ; Bolton, 2003). Un homme de haut rang se devait d'aider l'une de ses épouses ou de ses filles à acquérir un statut élevé, ce qui, selon Rivers, donnait lieu à une cérémonie kolekole ayant pour objet central la maison de la femme ainsi distinguée 5 . Cette dernière recevait aussi des tatouages sur l'ensemble du corps et des droits sur certains ornements tels que des ceintures en fibres de pandanus tressées et teintes (Rivers, 1914 : 131133). De manière générale, les très hauts gradés, hommes et femmes, possédaient une autorité qui pouvait s'étendre sur un territoire très large et qui, donc, transcendait les divisions locales (Vienne 1984: 186). Grâce à leur mariage notamment, les femmes de haut rang établissaient des liens d'alliance entre les différentes îles de l'archipel (Lanouguère-Bruneau, 2002: 347). Elles sont décrites à Mere Lava comme les ancêtres des lignages matrilinéaires.

Ce système d'acquisition de statut est aussi dépeint dans la littérature ancienne et les histoires orales recueillies à Mere Lava ${ }^{6}$ comme étroitement lié à l'organisation spatiale et résidentielle. Sur cette dernière île, à l'image des autres îles des Banks, la hiérarchie du système statutaire était matérialisée dans l'espace par les maisons des hommes, ne-gemel (Mwe), et leurs places de danse, ne-sere (Mwe). Associées aux matrilignages et situées dans les hameaux, les maisons des hommes étaient anciennement divisées en sections internes correspondant aux différents grades obtenus par les hommes du lignage. Elles étaient construites sur un plan rectangulaire et couvertes par une superposition de tuiles de feuilles de palmiers sagoutier qui s'étendait presque jusqu'au sol, lui-même surélevé sur une plateforme de pierre. Leurs toitures étaient soutenues par trois séries longitudinales de poteaux en troncs de fougère arborescente, l'ensemble des éléments de leurs structures étant fixé grâce à des lianes locales, na-garias (Mwe), selon un tressage dont les motifs étaient spécifiques aux lignages. Une ouverture permettait d'entrer par l'arrière de ces constructions pour les plus hauts-gradés. Ces maisons constituaient des espaces exclusivement masculins où les hommes se réunissaient pour discuter des affaires coutumières, transmettre des connaissances spécifiques aux jeunes générations, notamment lors des temps de réclusion préalables aux prises de grades. Ils étaient donc différenciés des maisons elles-mêmes, associées aux femmes. Celles-ci pouvaient être de deux types: soit des constructions de plain-pied obtenues selon les même techniques que les maisons des hommes mais de plus petite taille, avec une seule porte au seuil surélevé de façon à empêcher les cochons d'entrer et une façade de bois ou de roseaux tressés; soit des constructions surélevées sur des poteaux de bois prenant appui dans la pente de l'̂̀le, avec un plancher de lattes de bambou tressées. Les maisons des femmes de haut rang étaient néanmoins distinguées par un décor de motifs associés à leurs tatouages. Il ne semble pas qu'il y ait jamais eu à Mere Lava de construction spécifique destinée à accueillir les femmes lors de leurs menstrues mais la séparation des maisons dites « des femmes » et des ne-gemel (Mwe) pourvoyait sans aucun doute à l'isolement nécessaire. Les sociétés de danse, quant à elles, étaient liées à un espace entouré d'interdits situé à l'extérieur des villages appelé na-salagor (Mwe) où se transmettaient les connaissances et les techniques relatives aux danses masquées. Des constructions spéciales nommées elles aussi ne-gemel (Mwe) pouvaient y

4. Le concept de mana et sa signification ont été largement débattus dans la littérature académique. J'adopte ici la définition qui en est donnée par Thorgeir Kolshus pour l'île de Mota, dans le groupe des Banks : le terme mana est associé avec l'idée d'un pouvoir magique surnaturel et spirituel. Il correspond au verbe manag, employé pour parler de l'action de doter un objet ou un phénomène de mana (Kolshus, 1999: 154, voir aussi Kolshus 2013).

5. Voir cependant les travaux de Kolshus (2013 : 161-164) sur les limites de l'analyse du système statutaire ancien des îles Banks par William Halse Rivers. Celui-ci fonde son étude sur des données recueillies auprès d'un seul informateur principal, John Pantutun, élève de la mission anglicane puis catéchumène. Le rôle central des maisons dans l'obtention des statuts féminins pour les systèmes statutaires anciens de Mere Lava a néanmoins été confirmé par plusieurs interlocuteurs sur le terrain en 2010-2011 (voir aussi Lanouguère-Bruneau, 2002).

6. La description suivante est élaborée à partir de plusieurs entretiens menés entre 2010 et 2012, en particulier avec Adam Valuwa, Philip Gen, Leo Swithun, Clementine Mat, Lois Matias, Matias Rang, Jif John Norman Turris, Jif Luc Wokot et Jif William Sal, que je remercie pour ces informations. 
être réalisées selon le même plan que les ne-gemel des hameaux mais clos par une façade ornée de décors peints.

Cette description très générale permet d'avoir une première idée du système et de son fonctionnement. Pourtant, selon le contexte sociopolitique du temps, le nombre des grades ou des sociétés de danse était variable de même que leurs noms, les motifs et les insignes associés. Le déroulement exact des rituels d'acquisition des titres ou des droits dans les sociétés de danse fluctuait aussi. L'anthropologue Bernard Vienne, travaillant dans les îles Banks centrales dans les années 1970, note ainsi la grande plasticité de l'ensemble du système ancien dans une analyse qui peut sans aucun doute s'appliquer à Mere Lava :

«[...] si l'ordre des termes du Sukwe présente une congruence et une permanence certaine, leur développement quantitatif est extrêmement variable, non seulement d'une île à l'autre, mais dans la même île [...]. Nous faisons l'hypothèse, que cette variation relève de la dynamique des conjonctures démographiques et politiques et qu'ainsi les termes de la hiérarchie auraient tendance à se regrouper - c'est-à-dire pour certains à disparaitre - ou à se dédoubler selon la taille des groupes locaux et la densité et l'extension des réseaux de relations intergroupes. » (Vienne, $1971: 25-26$ )

L'architecture et l'organisation spatiale et résidentielle traduisaient étroitement cette variabilité ainsi que les tensions de l'ordre social au niveau des groupes locaux. Partout dans les îles Banks, les maisons des hommes étaient régulièrement transformées, de nouvelles maisons construites, des sections ajoutées ou laissées à l'abandon en fonction de la présence d'un homme de rang correspondant (Vienne, 1984 : 318). L'organisation spatiale générale dépendait donc de la formation de groupes de taille changeante en fonction de l'étendue de l'autorité des hommes de haut rang sous laquelle ils se plaçaient. L'autorité de ces hommes s'inscrivait alors physiquement dans le territoire, où elle était matérialisée par l'emplacement des divers ne-gemel (Mwe) et des résidences (Vienne, 1984 : 132). L'architecture révèle aussi le fait que les stratégies individuelles d'acquisition de prestige jouaient sur l'intégration d'éléments exogènes et la valorisation de ceux-ci. Certains travailleurs employés sur les plantations de Fidji ou du Queensland ont ainsi mis en valeur leur expérience après leur retour dans leurs îles. L'histoire orale du hameau de Tolo sur l'île de Gaua garde par exemple la mémoire d'un certain Vagal et de sa maison des hommes (nakamal), construite dans la deuxième moitié du XIX ${ }^{\mathrm{e}}$ siècle de façon à res- sembler à la maison principale de la plantation du Queensland sur laquelle il avait travaillé (Salatiel Vavak, Gaua, 27/09/2012). De même, le missionnaire Charles H. Brooke garde la mémoire de William Qasfor, de l'îlot Ra, proche de Mota Lava, qui construisit une maison des hommes sur le modèle de la maison du Commodore à Auckland (Brooke, 1873 : 600), démontrant cette fois une influence des voyages initiés par l'arrivée dans la région de la mission anglicane.

\section{Influence de la Melanesian Mission}

Dans la seconde moitié du XIX ${ }^{e}$ siècle, l'arrivée des missionnaires anglicans de la Melanesian Mission influence durablement l'organisation spatiale dans la région des îles Banks. En 1848, l'évêque de Nouvelle-Zélande George Augustus Selwyn organise un premier tour des îles de Mélanésie. L'objectif est d'avoir un premier contact avec les insulaires et, si possible, de prendre à bord quelques jeunes gens qui pourraient être éduqués dans la foi chrétienne au St John College à Auckland. Face à un contexte où la diversité des langues parlées et le climat rendent difficile une approche par les missionnaires européens, Selwyn attend de ces jeunes gens qu'ils soient ensuite les instruments de la conversion de leurs îles respectives. À partir de cette date, il organise deux à trois voyages par an, de façon à ce que les convertis passent les mois d'été à l'école de la mission et les mois d'hiver dans leurs îles. Les premiers voyages anglicans se concentrent au sud de l'archipel des Nouvelles-Hébrides ${ }^{7}$ et sur les îles Loyauté, en Nouvelle-Calédonie. Ce n'est qu'à partir de 1856 que le bateau de la mission, le Southern Cross, atteint les îles Banks (Melanesian Mission, 1869: 83, 122). Les missionnaires visitent alors les îles de Mota, Vanua Lava et Ureparapara. Impressionné par le "caractère aimable» des populations, l'évêque Selwyn prend rapidement la décision de faire de Mota le lieu de l'école qu'il souhaite établir dans les îles pendant les mois d'hiver (Armstrong, 1900 : 48). La première station anglicane est ainsi établie sur cette île en 1860 et le Mota devient la langue générale de la mission. Dans les vingt années qui suivent, la présence missionnaire anglicane s'installe durablement sur toutes les îles des Banks. Cependant, il s'agit au départ d'une présence discontinue puisque les missionnaires ne passent que quelques mois par an dans les îles (Armstrong, 1900: 102).

À Mere Lava, il semble que les contacts se limitent d'abord au rivage situé au niveau du village de Tasmat ${ }^{8}$. L'histoire orale de l'île garde,

7. L'archipel du Vanuatu conserve le nom de Nouvelles-Hébrides donné par James Cook en 1774 jusqu’à son Indépendance en 1980 où il est renommé Vanuatu, " Pays-qui-se-tient-debout ».

8. Bien que dès 1860 , les rapports consignent quatre jeunes garçons originaires de Mere Lava (Melanesian Mission 1863 : 12) comme ayant déjà passé quelques mois au St John's College à Auckland, c'est en 1863 que le révérend John Coleridge Patteson se rend dans les villages surmontant le rivage (Codrington et Patteson, 1863 : 15). 
quant à elle, la date de 1857 pour l'arrivée du révérend John Coleridge Patteson. Elle raconte comment l'un des deux hommes de haut rang qui se partageaient alors l'autorité sur l'île, Qoqo, eu le dessus sur son rival Ringo pour accueillir le missionnaire et, ainsi, lui éviter la mort. Après l'avoir présenté aux esprits de l'île afin qu'ils l'accueillent comme un des leurs sans lui faire de mal, le haut-gradé Qoqo mena Patteson dans son hameau de Leurok et dans son ne-gemel (Mwe). Le révérend Patteson y resta trois jours, prêchant la parole chrétienne avant de repartir de l'île en emmenant deux des fils de Qoqo pour les éduquer à Auckland. Ce fut le début de la mission à Mere Lava. L'histoire raconte encore comment certains des élèves de la mission revinrent ensuite dans leur île et convertirent progressivement l'ensemble de la population jusquà ce que, au tournant $\mathrm{du} \mathrm{Xx}^{\mathrm{e}}$ siècle, la conversion de Qoqo lui-même vint la compléter. À ce moment, il est dit que Qoqo, qui avait déjà tué sept fois des cochons dans le cadre du système statutaire et ainsi obtenu le rang de no-wotok (Mwe), tua de nouveau des cochons, cette fois pour devenir chrétien (Deacon Steve Turris, 14/02/2011 ; voir Durand, 2014 : 278 pour l'histoire complète).

Au-delà de son intérêt local, cette histoire montre l'insistance des interlocuteurs contemporains sur une certaine continuité qui aurait existé à l'époque du contact et de la conversion entre le système statutaire et la religion chrétienne (mais voir Eriksen 2008 pour une vision inverse sur l'île d'Ambrym). Cependant, cette histoire évoque aussi lorsqu'on la croise avec des sources telles que les journaux des missionnaires, plusieurs éléments qui semblent caractéristiques de cette période ancienne. En tant que créateurs de liens sociaux extra-locaux et acteurs majeurs de la circulation des biens et des connaissances entre les îles, les hommes et femmes de haut rang furent probablement les interlocuteurs privilégiés des missionnaires. Le bon ou mauvais accueil réservé à ceux-ci s'inscrivit donc, pour une part importante, dans le cadre des stratégies d'acquisition de prestige et d'autorité au sein du système statutaire. C'est ce que semble suggérer ici la rivalité entre Qoqo et Ringo à l'arrivée du révérend Patteson sur Mere Lava. Par ailleurs, en dehors des bâtiments de leur résidence, les missionnaires diffusèrent l'enseignement chrétien dans les maisons des hommes et les lieux associés au système statutaire. À Mere Lava, le révérend Patteson passa trois jours à prêcher dans le ne-gemel (Mwe) du hameau de Leurok. Celui-ci devint ensuite le premier lieu d'implantation de l'école et de l’Église anglicane dans l'île.
De même, le journal du missionnaire Robert Henry Codrington révèle ses fréquentes visites dans les maisons des hommes des villages de Mota lors de ses séjours sur l'île ainsi que dans le na-salagoro (Mo), l'espace des sociétés de danse tamate (Mo) :

"In the afternoon I went to Takelvarea and sitting in the gamal among the elders, with the intention of discoursing profitably [...]. Afterwards I bought a piece of obsidian, a knife of wood and a needle made of a ray's tail spike and return carrying a qeta or arum root for dinner. After resting a little, [...], I went across the ravine to Maligo and stayed a little while in the salagoro but couldn't talk much for coughing. " (Codrington, 1870 : entrée du 8 septembre)

"[...] starting for Luwai at 1 o'clock. Arrived there I spent an hour in the gamal at Mana where they assured me that they were quite enlightened, had done away with all bad customs and were just like us. I made an attempt to show that they were not, but without apparent success. " (Codrington, 1870 : entrée du 11 septembre)

Certains des premiers élèves emmenés pour être éduqués à la foi chrétienne sont les fils des hauts gradés qui accueillent les missionnaires. À Mere Lava, ce sont les deux fils du haut gradé Qoqo qui partirent pour quelques mois à l'école de la mission à Auckland. Lorsqu'ils revinrent sur leurs îles, ces jeunes gens mirent à profit leur position statutaire pour transmettre les enseignements et les valeurs chrétiennes mais il semble aussi que parallèlement, ils aient également utilisé leurs expériences outre-mer pour augmenter leur prestige personnel au sein de la société insulaire et du système statutaire. Ce fut le cas de Georges Sarawia de Vanua Lava, l'un des premiers jeunes hommes embarqués sur le navire de la mission, le Southern Cross et qui devint très influent à Mota où il dirigea la station missionnaire pendant le dernier quart du XIX ${ }^{\mathrm{e}}$ siècle. Codrington note ainsi dans son journal les moments de réclusion passés par " George " dans le na-salagoro (Mo) (Codrington, 1870 : entrée du 9 septembre) et le missionnaire Walter John Durrad remarque les critiques dont il fut l'objet au début du $\mathrm{Xx}^{\mathrm{e}}$ siècle pour avoir reçu plus d'honneurs sur Mota en raison de son haut rang dans la hiérarchie du suqe (Mo) qu'en raison de sa position dans l'Église chrétienne (Samson, 2009: 68). Effectivement dès 1875, Codrington s'étonne de l'ampleur et de la rapidité de la conversion sur Mota, qu'il associe néanmoins au charisme de George Sarawia (Samson, 2009 : 65). On voit donc ici à quel point il serait faux de penser les changements liés à l'influence chrétienne comme imposés et unidirectionnels. Ils ont plutôt relevé de stratégies négociées au niveau inter-individuel par certaines personnes en position d'auto-

9. Il entre au service de la Melanesian Mission en avril 1899 et le quitte en juin 1913.

10. Nommé évêque de Mélanésie en 1894, il succède à cette position à John Augustus Selwyn. Il reste au service de la Melanesian Mission jusqu'en 1911. 


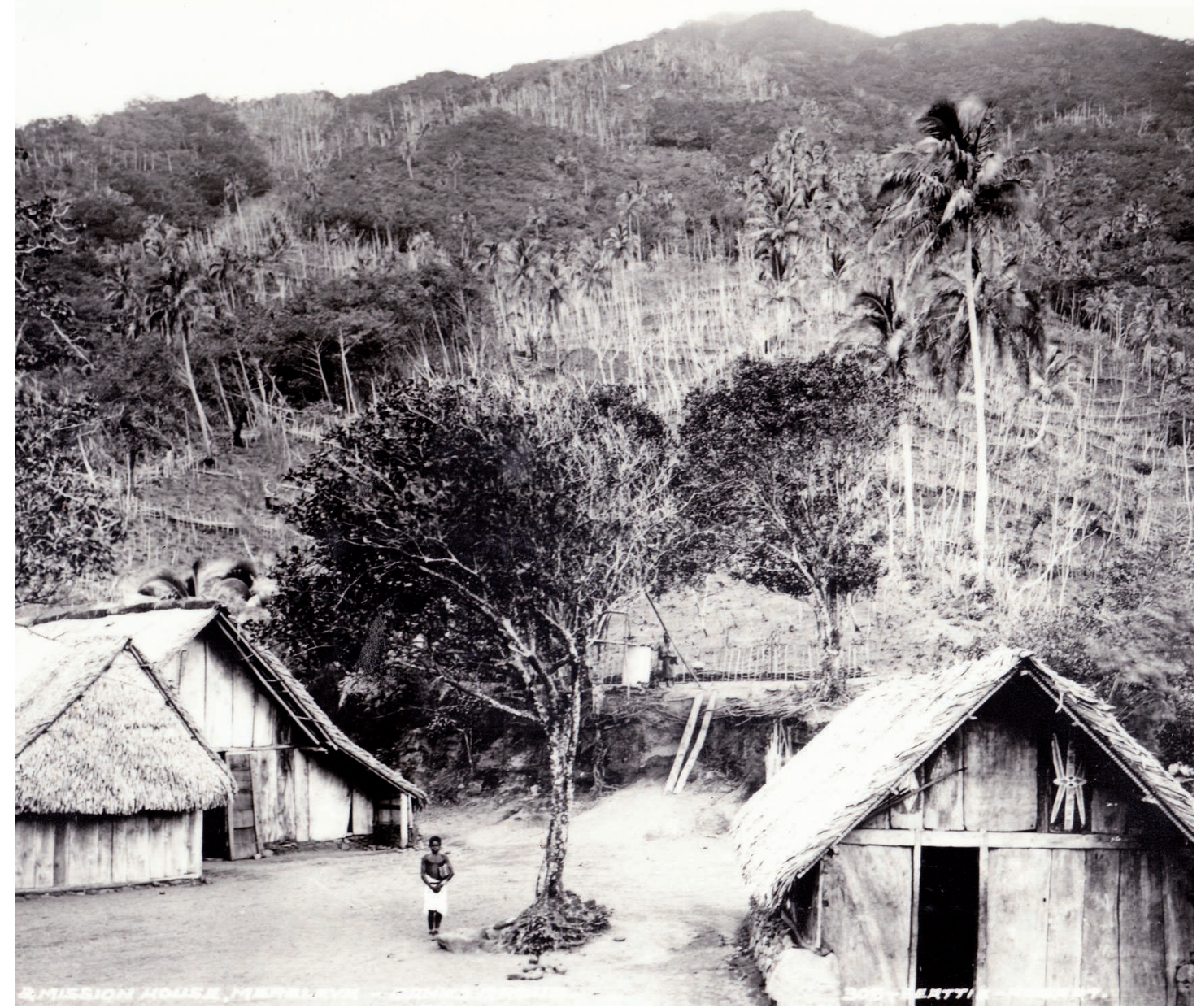

Figure 2. - Église et maison de la mission à Mere Lava, photo de John Watt Beattie, 1906 (@ Vanuatu Kaljoral Senta)

rité tels George Sarawia, l'évêque Patteson, Qoqo, etc. Ces transformations s'inscrivent dans le cadre dynamique du jeu de l'autorité et du pouvoir au sein du système statutaire local et possèdent donc un ancrage spatial important.

L'inscription dans le territoire est d'ailleurs une priorité pour les missionnaires et les teachers qui s'installent dans les îles. Le révérend Arthur Innes Hopkins note dans un recueil d'instruction publié en 1927 :

"The first need of the pioneer is somewhere to camp. His hosts have already, or soon will, put up a native hut to start with. There he will bestow himself and his boxes, and perhaps for a while his teachers. [...]. The first step then is to make friends. The friends of the people who received you will want a visit. So you collect a boat's crew, or a few carriers, and go off to some neighbouring village in the bush or on the coast. [...]. If you go to a friend's friend you will be well received. You will be fed and sheltered. [...]. That visit will lead on to a visit to another place, accompanied by some of your latest frien$d s$ who have access there. [...]. If the chief is friendly, food is brought to you, and you know then that you are welcome, and the place open for intercourse. That inter- course may lead to the acceptance later on of teachers, and the adoption of the new teaching. And a "school" is begun on a new site to which a few come to build huts, make gardens, put up a small first Church-school and so begin a "school" village. " (Hopkins, 1927 : 41-42)

Les bâtiments de la mission viennent donc s'ajouter aux maisons des hommes et aux na-salagoro $(\mathrm{Mo})$ comme lieu d'exercice de l'autorité à la fin du XIX ${ }^{e}$ siècle. Ils s'inscrivent dans un nouvel ensemble d'espaces matérialisant la présence de formes d'autorité plus multiples, qui intègrent l'enseignement chrétien comme l'une des voies permettant d'acquérir du statut. C'est sans doute dans ce contexte qu'il faut comprendre l'application de motifs associés aux esprits tamate (Mo) sur nombre de bâtiments associés à la mission. À Mere Lava, une photographie de John Watt Beattie prise en 1906 montre aussi ce type de motifs appliqués sur la mission house de la station anglicane du village de Tasmat (figure 2).

Dans un premier temps, la mission anglicane elle-même ne cherche pas à transformer fondamentalement la société insulaire. L'évêque George Augustus Selwyn note en 1847 : 
"[...] the aim of all missions should be to show that all Christ's religion is adapted to the circumstances and customs of all nations and every clime, and no established habits should be interfered with, unless they are directly contrary to the declared will of God. " (Melanesian Mission Occasional Papers, 1847 : 22, cité dans Hume, $1986: 306)$.

Si certaines pratiques sont réprouvées (Kolshus, 2014 : 159), Selwyn se donne néanmoins pour objectif de conserver "as much as possible of the traditional, social and cultural order as the basis of a Melanesian Christian Church" (Melanesian Mission Occasional Papers, 1847: 194, in Hume, 1986 : 306). Les missionnaires sont en fait confiants dans la capacité de l'enseignement chrétien à provoquer de lui-même l'abandon de pratiques coutumières qui ne sont, pour la plupart, pas perçues comme de nature religieuse. En 1870, Codrington écrit par exemple :

"I was visited by Siplaglano, with whom I had a long chat. The subjects were chiefly worldly. He is the only image maker in the island, others make them now, but as he says not the real things. As I never saw any thing except ornamental door posts, I could not accuse him of making idols. " (Codrington, 1870 : entrée du 7 septembre)

La situation se renverse au tournant du siècle. Les premières décennies du $\mathrm{xx}^{\mathrm{e}}$ siècle sont en effet marquées, aux yeux des missionnaires, par un désintéressement relatif des insulaires des Banks pour la mission et par un retour vers les pratiques coutumières associées au suqe (Mo) et aux sociétés tamate (Mo) (Annual Report of the Melanesian Mission, 1905). Le révérend H. V. Adams ${ }^{9}$ s'installe à partir de novembre 1902 sur l'île de Mota Lava pour un séjour qui ne se limite plus aux mois d'hiver. Il s'insurge rapidement contre le temps consacré par les insulaires aux affaires liées au système statutaire et s'oppose notamment aux divers interdits et réclusions cérémonielles qui viennent perturber la participation générale des individus à l'enseignement et à la vie de la mission (Durrad, 1920 : 2). L'évêque Cecil Wilson ${ }^{10}$ voit lui aussi dans le suqe (Mo) une menace pour la conversion et la pratique chrétienne. En 1904, les deux hommes organisent une réunion avec les insulaires sur l'île de Mota, à l'issue de laquelle il est décidé de réduire le temps consacré aux cérémonies liées au suqe $(\mathrm{Mo})$ à une journée :

"They pleaded that they were all in debt, and that only by circulation of money by means of suqe and other feasts, could they pay them off. They said that a general forgiveness of debts was impossible. Equally impossible was a general payment, for sufficient native money did not exist to pay all debts. Whenever a feast was made the giver of it gave away immense quantities of money, and some of this reached everyone, and thus debts could be paid, but they forgot that the unfortunate giver beggared himself by his generosity, borrowing, in order to give to others, at the exorbitant interest of 100 per cent. However the people held that they could be honest only by retaining the suqe, and so we then agreed that its meetings, with what was evil in them left out, should be permitted, but that they should last but one day at a time, and not for forty or fifty as previously, and that if this "sone-day rule» were broken, the people would give up the customs altogether." (Wilson, 1911 : 148-149)

Devant les faibles résultats donnés par cette mesure, un deuxième "meeting" est convoqué en 1909 . Le suqe (Mo) y est alors aboli pour deux ans, à l'issu desquels une nouvelle concertation est organisée, placée cette fois sous l'autorité de l'évêque Cecil E. Wood ${ }^{11}$. Cette dernière donne lieu à des prises de positions plus mesurées. Le suqe (Mo) est toléré mais doit être encadré par des principes chrétiens (Durrad, 1920 : 4).

Au début du $\mathrm{Xx}^{\mathrm{e}}$ siècle, les maisons des hommes (nakamal) et certaines pratiques anciennes persistent donc en même temps que les rythmes de vie et les figures de l'autorité se transforment (Vienne, 1971 : 57). L'espace résidentiel change en conséquence. Les villages chrétiens se développent et rassemblent désormais les maisons d'habitation autour d'une place centrale où se trouvent l'église et l'école de la mission.

L'unité de la maisonnée chrétienne, abritant ensemble le couple et ses enfants est encouragée. Les missionnaires s'opposent en particulier à l'une des pratiques associée au système statutaire : la séparation des feux de cuisson et le nonpartage de la nourriture entre personnes de statuts différents ainsi qu'entre hommes et femmes. Durrad remarque par exemple :

"Another count against the Suqe where it is carried out in its completeness, as in heathen places, is its antisocial character. It is antagonistic to the Christian idea that the family is the unit of society, and that family life is the best and highest kind of life. The Suqe takes no account of women otherwise than to secure their exclusion. A man and his wife do not share a common meal. This is anti-Christian. " (Durrad, $1920: 18$ )

Perçue comme incompatible avec la vie de famille chrétienne, la séparation des hommes et des femmes tout comme la hiérarchie des feux de cuisson est combattue ${ }^{12}$. À Mere Lava, Florence Coombe (1911 : 46) remarque l'introduction du

11. Nommé évêque de Mélanésie en 1912 et occupe cette fonction jusqu’en 1918.

12. Dans les îles Torres, situées au nord des îles Banks, cette volonté donne même lieu à une certaine «inversion " des pratiques de manière à rendre possible cette commensalité. Le catéchumène Léonard P. Robins informe ainsi l'évêque Wilson lors de son premier voyage dans les îles en 1894 sur les mesures prises dans ce sens : certains hauts-gradés mirent en place une série de repas dans les sections successives de moindre importance des maisons des hommes afin de faire dans le sens contraire le chemin qu'ils avaient fait en obtenant leurs grades (Wilson, $1895: 7$; voir aussi Samson, 2009: 80). 


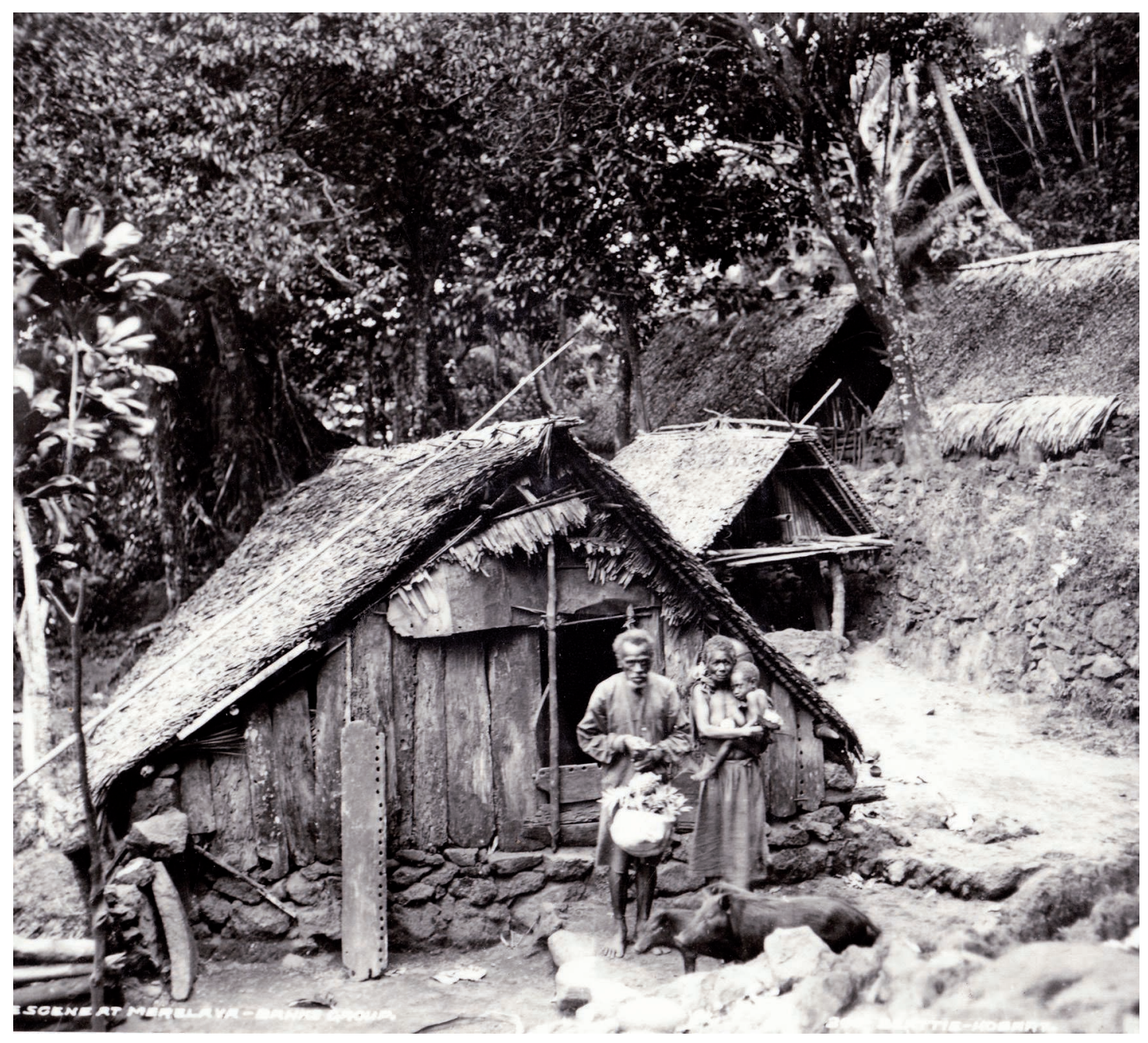

FiguRe 3. - Couple devant une maison, village de Tasmat, Mere Lava, photographie de John Watt Beattie, 1906 (C) Vanuatu Kaljoral Senta)

repas communautaire, partagé par les hommes et les femmes à la suite de la liturgie les dimanches.

À la même époque, les photographies de John Watt Beattie ${ }^{13}$ témoignent, pour le village de Tasmat, de l'autorité grandissante de la mission anglicane et de ses représentants. Face à la maison des hommes ne-gemel (Mwe), sur ce qui est toujours aujourd'hui la place centrale Sere (Mwe), et la surplombant, se trouvent l'église et la mission house (figure 2). Une autre photographie montre le nouvel ordre social préconisé : un couple pose devant sa maison, la femme tient dans ses bras un enfant et l'homme porte un panier rempli des produits de leur jardin (figure 3 ).

À ces transformations de l'architecture et de la société de la fin du XIX ${ }^{e}$ siècle et du début du $\mathrm{Xx}^{\mathrm{e}}$ siècle vont venir s'ajouter pendant les décennies suivantes celles dues à l'installation de l'autorité coloniale sur l'archipel.

\section{Politiques sanitaires et administration coloniale (1906-1980)}

\section{Politiques sanitaires et regard colonial: le contrôle des populations et la transformation de l'espace domestique}

Dès 1878 , face à la montée des revendications des ressortissants français et anglo-saxons installés dans l'archipel et aux tensions grandissantes créées par les demandes de protection répétées qu'ils adressent à leurs gouvernements respectifs, la France et la Grande-Bretagne s'engagent l'une et l'autre à ne pas annexer les Nouvelles-

13. John Watt Beattie (1859-1930) est un photographe et antiquaire installé à Hobart en Tasmanie. En 1906, il accompagne les missionnaires de la Melanesian Mission dans l'un de leurs voyages vers les îles du Pacifique Ouest et en rapporte un ensemble important de photographies couvrant l'ensemble des régions visitées.

14. Archives nationales d'Outre-Mer, Fonds Ministériel (FM), Série Géographique (SG) : NHB//1.A1(1). Copies de la lettre de l'ambassadeur de France à Londres, le Marquis d'Harcourt, au secrétaire d'Etat aux Affaires étrangères britannique, 
Hébrides ${ }^{14}$. De son côté, la France continue néanmoins à évaluer l'intérêt commercial et stratégique d'une telle annexion comme en témoigne la mission du contre-amiral Bergasse du PetitThouars, mandaté en 1879 pour explorer les îles principales de l'archipel ${ }^{15}$. En 1887, les gouvernements français et britannique établissent une Commission navale mixte chargée de maintenir l'ordre dans la région, puis signent en 1906 une Convention commune afin de "mettre fin aux difficultés résultant de l'absence de juridiction sur les indigènes » et " de régler les différents fonciers de leurs ressortissants respectifs" (Protocol between Great Britain and France respecting the New Hebrides, 1906 : 425 ${ }^{16}$. L'archipel devient alors un Condominium franco-britannique, où ni l'une ni l'autre des deux puissances ne peut imposer sa souveraineté (MacClancy, 1981 : 80).

Deux commissaires-résidents sont en charge des intérêts respectifs de la France et de la GrandeBretagne. Certains services tels que la poste, les travaux publics, la police ou la santé publique sont communs aux deux pays. D'autres, tels que l'éducation, font l'objet d'administrations parallèles (Protocol between Great Britain and France respecting the New Hebrides, 1906 : 428-429). Dans la pratique, la coopération entre les deux parties s'avère complexe à mettre en place et la présence du gouvernement colonial est inégale selon les îles (Scarr, 1967 ; MacClancy, 1981).

Les îles Banks, quant à elles, sont relativement peu touchées par les politiques coloniales pendant toute la période du Condominium. Celles-ci se concentrent en grande partie sur la résolution des nombreux conflits fonciers et ne sont sans doute que peu concernées par cette région où de rares colons sont installés. Pendant toute la première moitié du $\mathrm{xx}^{\mathrm{e}}$ siècle, seuls quelques individus tentent leur chance dans la région (O'Reilly, 1957). La crise de 1929 et la chute consécutive dramatique des cours du coprah entraînent, de surcroit, le départ de la plupart de ceux qui s'y étaient aventurés dans les premières décennies du siècle. Edgar Aubert de la Rüe, géologue français en mission dans la région remarque ainsi, au milieu des années 1930, un unique colon installé à Vanua Lava (Aubert de la Rüe, $1945: 87)^{17}$. Malgré la présence à partir de 1911 de délégués du Condominium dans les îles (MacClancy, 1981 : 84), c'est principalement par le biais des missionnaires qu'est rendue effective la présence du gouvernement colonial. Hopkins précise en 1927 que les missionnaires, puisqu'ils peuvent se targuer d'une connaissance plus approfondie du terrain, se doivent d'être les go-between entre le gouvernement, les traders et les habitants afin de défendre au plus juste les intérêts des uns et des autres (Hopkins, 1927 : 34-35). Les missionnaires anglicans, soutenus par des financements du gouvernement britannique, prennent donc en charge les missions éducatives et sanitaires dans les îles où ils exercent leur influence. Comme le montre un diagramme publié dans un rapport sur l'avancement de la mission de 1949, éducation et santé forment avec l'évangélisation les trois piliers de l'œuvre missionnaire anglicane (Melanesian Mission, 1949 : 27) (figure 4).

Les politiques sanitaires sont aussi intimement inclues dans le projet colonial où elles apparaissent comme l'un des moyens d'imposition du contrôle de l'État sur le territoire et les populations (Widmer, 2008). Dès la mission de Bergasse du Petit-Thouars, en 1879, un médecin, le docteur Monin, est chargé d'un rapport sur l'état de salubrité de l'archipel et celui des populations. Nicholas Thomas (1990) montre, pour Fidji, qu'à la fin du XIx ${ }^{\mathrm{e}}$ siècle, la volonté d'évaluer les conditions sanitaires des populations et les propositions faites pour les améliorer sont intimement associées au souci impérialiste d'ordonner, de clarifier les espaces de vie (et les pratiques) des insulaires afin de créer des entités villageoises nettes et propres, accessibles, compréhensibles et contrôlables par l'État colonial. Â l'inverse, semble-t-il, Monin pointe :

"Le climat des Hébrides est [...] un climat malsain et cependant nous devons avouer que tous les indigènes que nous avons vu se portent bien et ne paraissent pas souffrir de la malaria. Cette immunité leur vient sans doute, en dehors de la question de la race, qui met les nègres plus à l'abri des émanations palustres, des soins qu'ils ont de ne se fixer que dans les parties sèches et hautes de leur île, de leur vie indolente et de l'absence de grands défrichements. Des blancs amenés à vivre dans le même milieu résisteraient peut être aussi et réussiraient à conserver longtemps leur santé s'ils se trouvaient dans des conditions analogues, c'est à dire s'ils adoptaient les mœurs, les habitudes, la paresse intellectuelle et physique des insulaires. Mais tel ne serait pas leur but. " ${ }^{18}$

le Comte de Derby, datée du 15 janvier 1878 et réponse de celui-ci, du 26 février 1878 dans lesquelles chacun exprime sa volonté de conserver les Nouvelles-Hébrides comme territoire indépendant.

15. Archives nationales d'Outre-Mer, FM, SG : NHB//1.A1(1). Copie de la lettre de mission de A. Pothuau, ministre de la Marine et des Colonies au Contre-Amiral Bergasse du Petit-Thouars, $1^{\text {er }}$ octobre 1878 et Rapport du Lieutenant de vaisseau Roberjot, secrétaire de l'Amiral Dupetit Thouars, sur les Nouvelles-Hébrides, adressé à la Société de géographie de Paris, 22 avril 1879.

16. Cette Convention commune, qui n'accordait aucune place légale aux insulaires, sera cependant modifiée afin de pouvoir prendre en compte les affaires « indigènes » par un Protocole, signé en 1914, mais ratifié seulement en 1922 (Mac Clancy, $1981: 84)$.

17. Il s'agit très probablement de l'Australien Tiby Hagen (O’Reilly, 1957 : 92).

18. Archives nationales d'Outre-Mer, FM, SG : NHB//1.A1(1). Rapport du docteur Monin, 1879. 


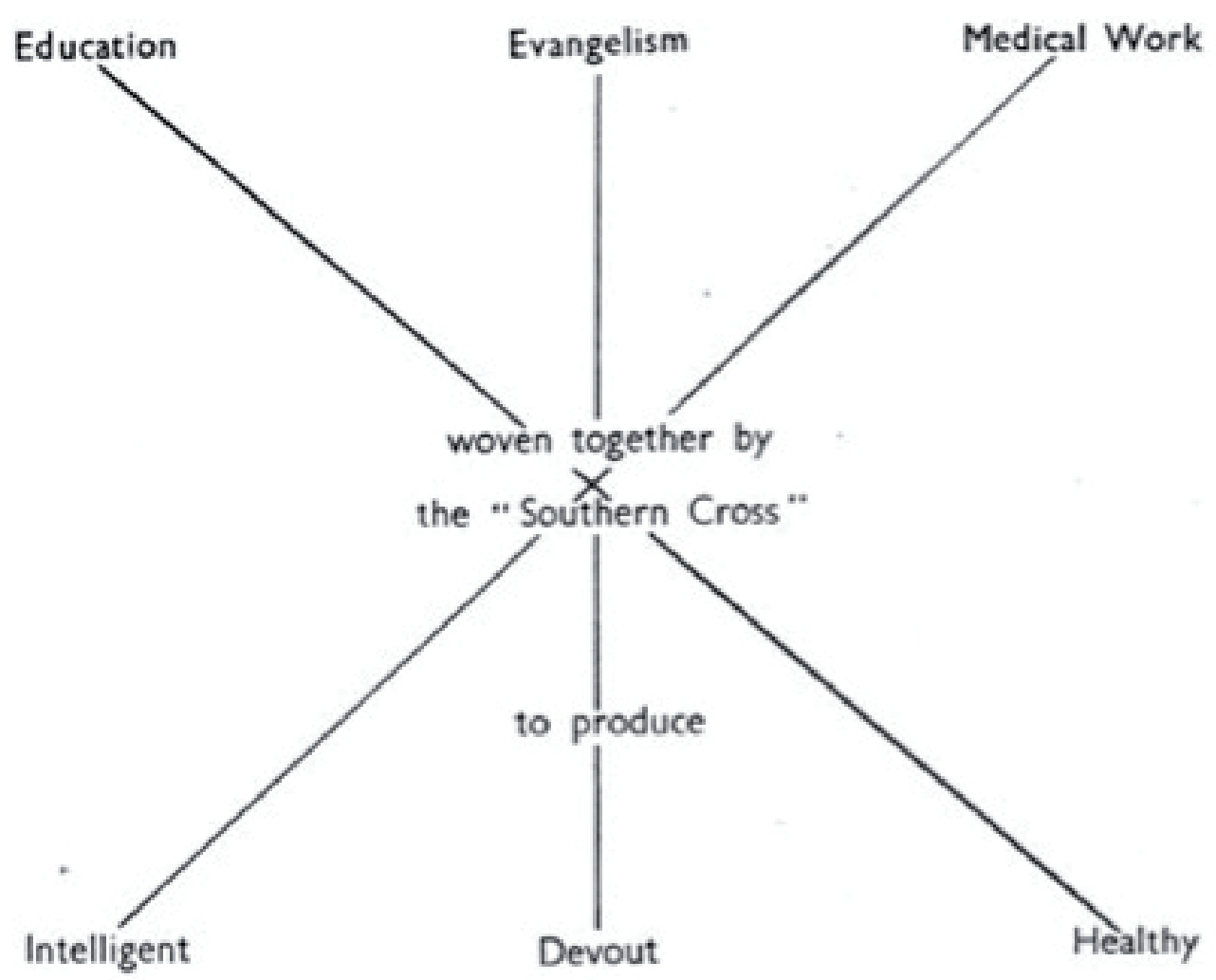

CHRISTIAN COMMUNITIES

Figure 4. - Diagramme publié dans Pacific Progress 1849-1949, "From the beginning, three strands have been inextricably interwoven in the pattern of Christian life created in Melanesia" (@) Project Canterbury, 2007)

Ces conclusions révèlent que l'intérêt pour la salubrité des espaces est aussi lié à l’appréhension de la maladie qui, jusque dans les premières décennies du $\mathrm{xx}^{\mathrm{e}}$ siècle, fait de l'environnement une cause majeure d'affection des corps (Curtin, 1989 ; Macchiusi, 1996 : 46-47) ${ }^{19}$. Les politiques publiques mises en place par les gouvernements français et britannique du Condominium, comme ailleurs dans le Pacifique, vont donc aussi concerner les corps qui ne sont pas - ou pas encore - malade en cherchant à traiter l'ensemble de l'environnement et de l'espace de vie (Macchiusi, 1996 : 48 ; Armstrong, 1983; Arnold, 1988 ; Thomas, 1990). Elles vont plus particulièrement se pencher sur l'espace de la vie quotidienne et les maisons d'habitation.

Une commission sanitaire est formée dans la capitale et en 1931, une réglementation conjointe définit précisément les conditions sanitaires qui doivent être respectées à Port Vila. Outre la régulation des nouvelles constructions à l'intérieur des limites de la capitale, le texte place aussi l'organisation interne des maisonnées sous la juridiction et le regard du gouvernement colonial. Il liste les conditions sanitaires de lumière et de ventilation à respecter et donne les dimensions des pièces et des fenêtres. Des inspecteurs sanitaires sont chargés de contrôler le respect de ces règles et les propriétaires privés sont mis en demeure d'effectuer les travaux d'assainissement nécessaires à leurs résidences sous deux ans (Condominium des Nouvelles-Hébrides, Réglementation conjointe $\mathrm{n}^{\circ} 6,1931$ ).

Cependant, si ces politiques sanitaires s'appliquent à la capitale et à sa population européenne ${ }^{20}$, leur application dans les îles et en milieu rural reste très peu documentée. Au regard de la faible présence gouvernementale dans certaines régions (comme les îles Banks) et de l'absence générale de financements, il est peu probable que ces politiques sanitaires aient eu un impact important dans tout l'archipel, même si la notion

19. À l'époque les fièvres sont par exemple populairement associées à la chaleur tropicale et les maladies pulmonaires à l'air stagnant (Curtin, 1989 ; Macchiusi, 1996: 47). 
de villages ordonnés et accessibles, placées sous le « regard » et offrant la possibilité d'un contrôle de l'administration (Thomas, 1990), trouve un relais dans l'ordre et les idées sanitaires prônées par les missionnaires ${ }^{21}$. En 1940, une autre réglementation conjointe donne cependant aux délégués du Condominium le pouvoir d'ordonner le nettoyage des espaces villageois dans les zones rurales ou encore leur évacuation et leur relocalisation pour des raisons sanitaires (Condominium des Nouvelles-Hébrides, Réglementation conjointe $\left.\mathrm{n}^{\circ} 12,1940\right)$. À partir de cette date, la présence administrative et sanitaire du gouvernement colonial est progressivement facilitée pour ces zones "de brousse ": des médecins insulaires sont envoyés pour être formés à Fidji (Bador, 1982 : 62). Les Colonial Development and Welfare Acts britanniques de 1940 et 1945 et l'obtention de financements pour des dépenses régulières de fonctionnement du Condominium (Overseas Development Institute, 1964 : 21-22) facilitent leur formation à l'étranger ainsi que celle d'infirmières, et permettent la création de dispensaires supplémentaires dans les îles (Bador, $1982: 62,71,81)$. Les politiques sanitaires se diffusent donc probablement plus largement dans les zones rurales à partir de cette époque. Ainsi, pour le nord de Pentecôte, l'histoire orale associe certains changements dans l'organisation spatiale des maisonnées avec les initiatives de développement sanitaire mises en place à partir de 1953 par le docteur Philip Ilo, médecin formé à Fidji et dirigeant le dispensaire d'Abwatuntora. $\mathrm{Ce}$ dernier encouragea en particulier la séparation des cuisines ou des lieux de préparation des repas et des maisons pour dormir, de façon à limiter le risque de mortalité dû aux incendies et à réduire les effets sur la santé de l'inhalation continuelle de fumée dans les maisons (Taylor 2008 : 144-145, voir aussi Bador, 1982 : 81). Dans les îles Banks, une clinique fut construite à Mota Lava en 1955 et dirigée par l'Assistant Medical Practitioner Stanley Reveaf (Bador, $1982: 81)$.

À Mere Lava, comme dans le nord de Pentecôte, les maisons-cuisine et les toilettes sont dits être d'introduction récente. Il semble cependant que les transformations de l'architecture y aient eu des résultats différents. En effet, si l'on trouve aujourd'hui, dans les deux cas, des maisons-cuisine bien différenciées des maisons pour dormir, alors qu'à Pentecôte, les premières peuvent être des abris de construction plus rudimentaire (Taylor, 2008 : 145), à Mere Lava, les cuisines sont généralement les constructions les plus élaborées et font l'objet de soins particuliers dans leur réalisation technique. Les cuisines de Mere Lava sont aussi données aujourd'hui comme des constructions coutumières, tandis que les maisons pour dormir sont considérées comme secondaires et comme lieux possibles d'innovations architecturales multiples. Autrement dit, les lieux de préparation de la nourriture auraient été sortis des maisons à Pentecôte, tandis qu’à Mere Lava, ce sont les lieux pour dormir qui en auraient été séparés.

Nous suggérons que ceci doit se comprendre en conjonction avec une autre transformation sociétale importante ayant eu lieu après la Seconde Guerre mondiale dans les îles Banks, à savoir l'abandon partiel des pratiques liées au système statutaire et, notamment, de la hiérarchie des grades.

\section{Institution des assesseurs et abandon de la hié- rarchie des grades dans les îles Banks}

Dans la première moitié du $\mathrm{Xx}^{\mathrm{e}}$ siècle, des actes de transgression sont commis dans le cadre de l'influence grandissante de l'idéologie chrétienne et de l'opposition des missionnaires au système statutaire. À Mere Lava par exemple, l'histoire orale retient comme un moment de rupture important l'entrée d'une femme de haut rang et de sa fille dans le ne-gemel (Mwe) du hameau de Leurok, au village de Tasmat.

En 2010-2011, cet acte de transgression était toujours conté comme l'instant où la hiérarchie des grades a commencé à péricliter et où certains des objets conservés dans les maisons des hommes ne-gemel (donc à l'abri du regard des enfants et des femmes) furent sortis de celles-ci pour être placés dans les maisons-cuisine, $n$-ean kuk. Il s'agit, en particulier, des objets de bois sculptés liés à la préparation du no-löt (pudding de tubercules écrasés dont la préparation et la consommation étaient réservées aux hommes) ou des paniers contenant la monnaie de coquillage utilisée dans les contextes cérémoniels.

Ces actes de transgression furent appuyés, après la Seconde Guerre mondiale, par la création coloniale de conseils régionaux (MacClancy, 1981 : 121 ; Lindstrom and White, 1997 : 213). Des assesseurs furent alors nommés dans les îles pour siéger au niveau régional au côté du Délégué du Condominium et le conseiller. À Mere Lava, la nomination d'un assesseur fut, selon les dires des interlocuteurs contemporains, ce qui a porté un coup fatal à la hiérarchie des grades. Là où l'autorité était placée entre les mains de quelques hommes de haut-rang dont les statuts étaient sujets à une compétition politique soute-

20. Le même texte réglemente aussi les mouvements de la population insulaire, interdite de résidence à l'intérieur des limites de la capitale (Condominium des Nouvelles-Hébrides, Réglementation Conjointe n6, 1931).

21. Des hôpitaux et cliniques sont néanmoins créés dans les centres administratifs et missionnaires dès le début du siècle. Pour un aperçu sur le développement de ceux-ci ainsi que sur le système de santé aux Nouvelles-Hébrides, voir Bador (1982). 
nue dans le contexte du système statutaire, l'administration coloniale désigna un seul assesseur dont l'autorité s'étendit sur l'ensemble de l'île. Un interlocuteur se rappelle ainsi :

"Tout a changé quand les deux gouvernements ont mis des assesseurs dans toutes les îles. Là, la coutume a commencé à disparaître, les hommes de haut rang sont passés au second plan tandis que les assesseurs venaient au premier. C'est arrivé dans les années 1960. C'est à ce moment-là que les chefs ont arrêté de prendre des rangs. Après, c'est l'assesseur qui s'est occupé de toute l'île. Les tueries de cochons se sont terminées à cette époque. » (Marsden Harris, Gaua, 18/09/2012, ma traduction du bislama)

Malgré la tentative de quelques uns, dans les années 1980 , de ressusciter les prises de grades (Deacon Steve Turris, Mere Lava, 05/01/2011) sur Mere Lava, l'organisation spatiale a été directement et durablement influencée par ces transformations. Les maisons des hommes situées dans les hameaux et associées aux matrilignages furent progressivement abandonnées comme lieu de l'autorité au profit d'une seule maison des hommes située sur la place centrale des villages. L'église et cet unique ne-gemel (Mwe) sont désormais les lieux de réunion où les affaires de la communauté villageoise sont discutées, souvent après la liturgie du dimanche.

La période coloniale vit donc l'organisation sociale dans l'île de Mere Lava passer graduellement de formes de l'autorité qui plaçaient la compétition au niveau des groupes locaux dispersés dans le territoire et des matrilignages, à d'autres formes de leadership, plus multiples, qui mettent l'accent sur les communautés villageoises ${ }^{22}$. Dans les hameaux, des maisons pour dormir n-ean- bë moёtur sont maintenant construites, et différenciées des maisons-cuisine n-ean ${ }^{-} k u k$ qui deviennent, elles, les constructions centrales. Au vu de cette histoire socio-architecturale, il est donc permis de questionner plus avant l'importance actuelle des maisons-cuisine et en particulier de la mettre en perspective avec le rôle qu'elles conservent par rapport aux matrilignages.

\section{Prestige, maisons-cuisine et maisons pour dormir contemporaines}

En 2010-2011, il y avait plusieurs figures de l'autorité sur Mere Lava. Dans chaque village, un ou plusieurs chefs étaient en charge de la gestion des événements de la communauté et des litiges, coutumiers ou non. Ces chefs se réunissaient régulièrement en un Conseil de l'île, présidé par un
Paramount Chief élu pour trois ans. Par ailleurs, un pasteur, des diacres et des catéchumènes assuraient la direction de la communauté religieuse, à majorité anglicane. Enfin, certains hommes possédaient aussi des positions prééminentes au sein du na-salagoro (Mwe), l'espace masculin des sociétés de danse, toujours actives sur l'île. Le plus souvent, un seul et même individu occupait plusieurs de ces positions, à l'instar du diacre du village de Tasmat, qui était aussi à la tête du nasalagoro de ce village ou du catéchumène étant aussi l'un des chefs de la communauté. Cette situation démontre l'imbrication étroite qui caractérise aujourd'hui les positions d'autorité au sein de l'Église, du gouvernement et de la coutume (Durrad, 1920; Allen, 1981 ; Vienne, 1984 ; Kolshus 1999; Lanouguère-Bruneau, 2002; Tabani, 2002 ; Hess, 2009).

Cette imbrication des formes de l'autorité se retrouvait aussi pour les femmes. L'une d'entre elles était désignée comme ne-ngweter (Mwe), nom anciennement donné aux femmes de haut rang, et élue à la tête du Conseil des femmes de l'île. Elle était de plus chargée de représenter les intérêts des femmes lors des réunions du Conseil des chefs de l'île. Choisie en général pour sa connaissance approfondie de la coutume, la femme tenant cette position était représentée dans chaque village par deux déléguées, respectivement présidentes et vice-présidentes des femmes du village. Enfin, un certain nombre de femmes obtenaient aussi un statut particulier grâce à leur position au sein du groupe religieux Mother's Union. Ces femmes dénommées veve (Mwe), " mères ", étaient considérées comme les relais de l'autorité de l'Église anglicane au sein des maisonnées, s'occupant principalement des problématiques concernant la santé, la famille et l'éducation des enfants. Dans la pratique, nombre de femmes cumulaient à la fois une position au sein du groupe Mother's Union et du Conseil des femmes.

Sur le plan architectural, il existait toujours en 2010-2011 à Mere Lava des lieux communautaires associés à l'autorité : l'église et la maison des hommes ne-gemel (Mwe) de la place centrale des villages ainsi que, parfois, une maison des femmes où se déroulaient les meetings et les activités des femmes. Dans les hameaux en revanche, ce sont les maisons-cuisine qui se trouvaient au centre de la vie quotidienne, mais aussi d'un certain nombre de moments cérémoniels dont les plus importants étaient ceux marquant la naissance et la mort.

C'est à l'intérieur des maisons-cuisine que les femmes donnaient naissance à leurs enfants lorsqu'elles n'avaient pas eu le temps ou pas voulu

22. L'histoire orale de l'île de Mere Lava raconte aussi par exemple comment, au moment de l'Indépendance, en 1980, ce ne sont pas les différentes matrilignages mais bien les deux villages de Tasmat et de Leqel qui se sont disputés l'obtention d'un bœuf, offert par le gouvernement pour contribuer aux célébrations dans l'île. 
aller au dispensaire. Les premiers rites attachant l'enfant à sa qualité de vivant et à son matrilignage étaient pratiqués à l'intérieur ou devant ce bâtiment. Il s'agissait de laver la bouche de l'enfant avec de l'eau salée afin de retirer les dernières traces de substances liées à la naissance, de le confier symboliquement à une sœur de mère et, par un don informel d'argent fait par les parents aux oncles maternels de l'enfant peu de temps après la naissance, de lui assurer l'enseignement et le soutien futur de ceux-ci. De même, la mort d'une personne survenait souvent dans ces maisonscuisine, puisque c'est à l'intérieur de ces maisons que les proches prenaient généralement soin des personnes âgées et des malades. La maison-cuisine associée au défunt était par ailleurs le centre des activités funéraires. Les multiples repas cérémoniels étaient préparés à l'intérieur et le rituel de compensation visant à transmettre les droits sur les terres et les ressources du mort à ses enfants avait lieu immédiatement devant ce bâtiment.

Sur l'île de Mere Lava, les maisons-cuisine sont donc aujourd'hui des lieux essentiels de la reproduction sociale, liées étroitement aux matrilignages et à leur prestige. La centralité de ces maisons, en terme sociologiques, s'exprime parfois par l'ajout d'ornements signalant le statut de leur commanditaire. Il s'agit par exemple d'une ligne de pierres marquant la façade, d'éclisses de bambou épointées ajoutées sur le toit ou de bambous coupés en pointe et aux bords dentelés scandant la bordure du fronton. Leurs structures et leurs emplacements dans l'espace des hameaux évoquent aussi pour certaines maisons-cuisine un lien plus direct avec l'architecture prestigieuse des anciennes maisons des hommes. Leur surélévation sur une plateforme soutenue par des murs de pierre et les trois rangées longitudinales de poteaux que présentent nombre d'entre elles sont ainsi des caractéristiques que les interlocuteurs attribuent aux anciennes maisons des hommes.

Enfin, elles matérialisent l'autorité et le prestige de leur commanditaire par l'ampleur de l'organisation technique et sociale nécessaire à leur construction. La maîtrise d'œuvre est confiée par le commanditaire à un homme du lignage de son père qui doit alors mettre sur pied une équipe de travailleurs réguliers, hommes et femmes. Outre ces derniers, certaines étapes importantes de la construction rassemblent les parents paternels et maternels du commanditaire pour des opérations techniques nécessitant le travail commun d'un grand nombre de personne : la fabrication des tuiles de palmes de sagoutier de la couverture et leur fixation sur la charpente par exemple. Ces grands rassemblements représentent aujourd'hui des temps importants d'actualisation des liens sociaux. Chacun, parent paternel ou maternel, apporte sa contribution à la nouvelle maison, sous forme de matériaux de construction ou de nourriture qui sera consommée par les tra- vailleurs. À ce moment, la maison et la maisonnée qu'elle est destinée à abriter, apparaissent comme le résultat productif de l'alliance entre deux matrilignages, ce qui est ensuite publiquement montré lors d'une cérémonie qui marque la fin de la construction. Cette cérémonie, nommée no-wol-ean" (Mwe), littéralement "payer la maison " correspond à une série de prestations par lesquelles le commanditaire récompense le contremaître et son équipe (paternels) ainsi que certains parents maternels dont l'aide a été particulièrement importante.

La maîtrise de l'ensemble du processus de construction et la magnificence de la fête finale sont des éléments qui participent du prestige actuel des hommes et des femmes. La maisoncuisine, une fois terminée, vient rappeler l'importance de la maisonnée qui l'a commandité et de son chef. Elle assure sa présence et celle du matrilignage du commanditaire dans le territoire. Elle matérialise également le lien à la coutume (kastom) de celui-ci. C'est la raison pour laquelle certains migrants, bien que résidant sur d'autres îles, prennent soin de faire construire ou reconstruire les maisons-cuisine dans leur hameau d'origine à Mere Lava même lorsqu'ils ne les utiliseront que très brièvement lors de séjours dans l'île. Construire une maison-cuisine relève de stratégies d'affirmation relationnelles qui privilégient le lien à la coutume et au territoire.

En parallèle, la construction de maisons pour dormir est l'occasion d'innovations architecturales multiples. Lorsqu'elles sont construites en matériaux importés tels que le béton et la tôle ondulée, elles participent de stratégies complémentaires d'acquisition de prestige qui mettent cette fois l'accent sur la réussite financière et l'intégration aux sphères économique et politique de l'État-nation. Ces maisons sont généralement commanditées par des personnes de Mere Lava installées dans les centres urbains. Un membre de leur matrilignage resté sur l'île est alors désigné pour habiter et prendre soin de la construction.

Ce type de maisons est souvent construit sur un laps de temps beaucoup plus long. L'opération dépend en effet des capacités financières de la famille, mais aussi des passages du bateau qui amène progressivement les matériaux nécessaires. Il est alors difficile de mobiliser une équipe de travail définie. De plus, leur construction ne donne pas lieu aux rassemblements familiaux marquant certaines étapes de la construction coutumière, puisque ces étapes sont associées à des actions techniques précises (telles que fabriquer les tuiles du toit, ou bien les fixer sur la charpente comme on l'a vu). L'appréciation de la coopération familiale est, dans ce cas, déplacée des étapes mêmes de la construction à celles de l'obtention des matériaux payés puis envoyés par bateau par la famille établie dans les centres urbains. 


\section{Conclusion}

Le déroulement précis de cette chronique socioarchitecturale montre comment l'espace domestique et les maisons sont aujourd'hui des sites essentiels pour l'expression et la construction d'appartenances multiples au Vanuatu. À Mere Lava, les maisons-cuisine participent du prestige des individus et de leur matrilignages. Elles continuent de matérialiser dans les hameaux le statut et l'autorité des « oncles maternels ». À travers l'utilisation possible d'autres types de matériaux et de techniques, les maisons pour dormir, en revanche, permettent d'affirmer les liens avec les parents - de plus en plus nombreux - résidant dans les centres urbains. Ces deux types de constructions participent de stratégies sociales complémentaires permettant aux individus d'affirmer leur position à la fois sur le plan de la coutume et sur celui de l'économie de marché.

L'importance actuelle des maisons-cuisine tient à un double mouvement initié à la fois par les politiques missionnaires et par la politique de l'État colonial. Cette importance démontre l'impact profond que ces politiques ont eu sur les modes d'habiter et les conceptions locales de l'espace social au Vanuatu, mais elle démontre aussi l'aspect très dynamique des réactions architecturales insulaires aux changements historiques, économiques et sociaux des $\mathrm{XIX}^{e}$ et $\mathrm{XX}^{e}$ siècles. Laspect matériel, formel, de ces maisons apparait comme un élément essentiel à travers lequel les individus pensent leurs rapports à ces changements. En cela, poteaux, poutres, tuiles de feuilles des toits, murs de pierres empilées ou de bambou tressés, ciment des sols et tôles ondulées, de même que les connaissances et savoirfaire techniques mobilisés font partie intégrante de la négociation quotidienne du politique dans les îles Banks. Ceci contribue, aujourd'hui comme hier, à conserver une grande plasticité à l'organisation politique et sociale.

\section{BIBLIOGRAPHIE}

Allen Michael, 1981. Vanuatu: Politics, Economy and Ritual in Island Melanesia, Sydney, Academic Press of Australia.

Annual Report of the Melanesian Mission FOR 1905, 1906, The Southern Cross Log 11, pp. 1-11 (http://anglicanhistory.org/oceania/ cwilson/report1905.html).

Armstrong E.S., 1900. The History of the Melanesian Mission, Londres, Isbister and Company Limited.

Armstrong David, 1983. The political anatomy of the body: medical knowledge in Britain in the twentieth century, Londres, Cambridge University Press.

Arnold David, 1988. Imperial medecine and indigenous societies, Manchester, Manchester University Press.

Aubert DE LA RüE Edgar, 1945. Les NouvellesHébrides, Illes de Cendres et de Corail, Montréal, Éditions de l'Arbre.

BADOr Jean-Luc, 1982. Histoire des services de santé publique aux Nouvelles-Hébrides de 1895 à 1979, thèse, Lyon, Université Claude Bernard.

Best Simon, 1984. Lakeba: The Prehistory of a Fijian island, PhD Thesis, Auckland, University of Auckland.

Bolton Lissant, 2003. Unfolding the Moon: Enacting women's kastom in Vanuatu, Honolulu, University of Hawai'i Press.

Bonnemaison Joel, 1996. La coutume ou les formes du pouvoir politique traditionnel au Vanuatu, in C. Kaufmann et al. (éds), Vanuatu Océanie, arts des îles de cendre et de corail, Paris, RMN, ORSTOM, pp. 212-229.

Bourdieu Pierre, 1972. Esquisse d'une théorie de la pratique précédée de trois études d'ethnologie kabyle, Genève, Librairie Droz.

Brooke Charles, i 873. Progress of the Melanesian Mission, Mission Life 4, pp. 440-448 (http://anglicanhistory.org/oceania/brooke_ progress1873.html).

Buchli Victor, 20I3. An anthropology of architecture, Londres, Bloomsbury.

Carsten Janet and Stephen Hugh-Jones (eds), 1995. About the house: Lévi-Strauss and beyond, Cambridge, New York, Cambridge University Press.

Codrington Robert H., 1870, 1872, 1875 and 1881. Journals, Londres, sOAS Archives, MM/ section 2/box 9. 2/9.

-, 1972 [1891]. The Melanesians: Studies in their Anthropology and Folklore, New York, Dover Publications.

Codrington, Robert H., Patteson, John C. 1863. Lecture on the Melanesian Mission together with the report and accounts of the mission (http://anglicanhistory.org/oceania/ codrington_lecture1863.pdf).

CoIfrier Christian, 1988. Traditional Architecture in Vanuatu, Suva, Institute of Pacific Studies, University of the South Pacific.

Coоmbe Florence, 1911. Islands of enchantment, Londres, Macmillan \& Co. 
CurTin Philip, 1989. Death by migration: Europe's encounter with the tropical world in the nineteenth century, Cambridge, Cambridge University Press.

Deacon Bernard, 1934. Malekula, a Vanishing People in the New-Hebrides, Londres, G. Routledge \& Sons Ltd.

Durand Marie, 2014. The materiality of the kitchen house: building, food and history on Mere Lava, northern Vanuatu, PhD Thesis, Norwich, University of East Anglia.

-, 2015 (7 septembre). Thinking through houses: the materiality of kitchen houses and histri on Mere Lava, Vanuatu, Journal of Material Culture (prépublication en ligne, DOI : $10.1177 / 1359183515601452)$.

Durrad Walter J., 1920. The attitude of the Church to the suqe, Norfolk Island, Melanesian Mission.

Eriksen A., 2008. Gender, Christianity and Change in Vanuatu: an analysis of social movements in North Ambrym, Aldershot, Burlington, Ashgate.

Fox James J. (ed.), 1993. Inside Austronesian Houses: perspectives on domestic design for living, Canberra, Australian National University, Department of Anthropology, Comparative Austronesian Project, Research School of the Pacific Studies.

Hess Sabine, 2009. Person and place: ideas, ideals and the practice of sociality on Vanua Lava, Vanuatu, New York, Berhahn Books.

Hilliard David, 1978. God's Gentlemen: A History of the Melanesian Mission 1849-1942, St Lucia, University of Queensland Press.

Hopkins Arthur I., 1927. Melanesia To-day, a study circle book, Londres, Society for promoting Christian knowledge.

Huffman Kirk, 1996. Échanges, liens culturels et droits d'auteurs. Leur importance dans les arts du Vanuatu, in C. Kaufmann et al.,(éds), Vanuatu Océanie, arts des îles de cendre et de corail, Paris, RMN, ORSTOM, pp. 190-203.

Hume Lynne, 1986. Church and custom on Maewo, Oceania 56, pp. 304-313.

Kembol Luke A., I976. Enga housing and Enga traditions, Port Moresby, Institute of Papua New Guinea Studies, Occasional paper 4.

KIrch Patrick V. and Douglas E. Yen, 1982. Tikopia: The Prehistory and Ecology of a Polynesian Outlier, Honolulu, Bishop Museum Press.

Kolshus Thorgeir, 1999. Purism, syncretism, symbiosis: cohabiting traditions on Mota, Banks Is- lands, Vanuatu, PhD Thesis, Oslo, Department of Social Anthropology, University of Oslo.

-, 2013. Codrington, Keesing, and Central Melanesian mana: Two Historic Trajectories of Polynesian Cultural Dissemination, Oceania 83, pp. 316-327.

—, 2014. A House upon Pacific Sand, W.H.R. Rivers and His 1908 Ethnographic Survey Work, in E. Hviding and C. Berg (eds), The Ethnographic Experiment: A.M. Hocart and W.H.R. Rivers in Island Melanesia, 1908, New York, Oxford, Berghahn Books, pp. 155-176.

Lanouguere-Bruneau Virginie, 2002. Le corps de l'igname et le sang de la noix de coco : le système social ancien de Vanua Lava, îles Banks, Vanuatu, thèse, Paris, EHEss.

LaYArd John, 1942. Stone Men of Malekula, London, Chatto \& Windus.

LÉvi-Strauss Claude, 1987. Anthropology and Myth: Lectures 1951-1982, Oxford, New York, Blackwell Publishers.

Linstrom Lamont and Geoffrey White (eds), 1997. Chiefs Today: Traditional Pacific Leadership and the Postcolonial State, Stanford, Stanford University Press.

MacClancy Jeremy, 1981. To kill a bird with two stones: A short history of Vanuatu, Port Vila, Vanuatu Cultural Centre.

Macchiusi Joseph, 1996. Fearing the unknown. British hygienic and sanitary policy in the colonial New Hebrides, 1900-1940, North York, York University.

Mayne A.J.C., 1982. Fever, squalor and vice: sanitation and social policy in Victorian Sydney, St Lucia, Queensland University Press.

Melanesian Mission, 1863. Report of the Melanesian Mission for the years 1861-1862, London, Rivingtons.

-, 1869. The Island Mission: Being a History of the Melanesian Mission from Its Commencement, Londres, William Macintosh.

-, 1949. Pacific Progress 1849-1949: being the illustrated centenary book of the Diocese of Melanesia, Londres, Parrett \& Neves.

Morgan Lewis Henry, 1881. Houses and house-life of the American aborigines, Washington, GPO.

OLIVER Paul, 2006. Built to meet needs, cultural issues in vernacular architecture, Oxford, Elsevier Ltd.

O’Reilly Patrick, 1957. Hébridais, répertoire biobibliographique des Nouvelles-Hébrides, Paris, Société des Océanistes, Publications de la Sdo 6. 
Overseas Development Institute, 1964. Colonial Development, a factual survey of the origins and history of British aid to developing countries, Londres, The Overseas development Institute Ltd.

PALMER John, 1866. Journal (http://anglicanhistory.org/oceania/palmer/journal1866.html).

Peltier Philippe, 2008. Men's houses, Other people's houses, in S. Tcherkézoff and F. Douaire-Marsaudon (eds), The Changing South Pacific, Identities and Transformations, Canberra, ANu E-Press, pp. 63-84.

Protocol between Great Britain and France respecting the New Hebrides, February 1906. (http://www.usp.ac.f//fileadmin/random_images/home_middle_banners/emalus/ Pacific_law_materials/Vanuatu/4.Protocol_ between_Great_Britain_and_France_respecting_the_new_Hebrides_February_1906. pdf., consulté le 29/12/2015).

Rapoport Amos, 1976. Pour une anthropologie de la maison, Paris, Dunod.

Rivers William H., 1914. The History of Melanesian Society, Cambridge, Cambridge University Press.

Rodman Margaret, 1985. Moving Houses: Residential Mobility and the Mobility of Residences in Longana, Vanuatu, American Anthropologist 87, pp. 56-72.

—, 1987. Masters of Tradition: Consequences of Customary Land Tenure in Longana, Vanuatu, Vancouver, University of British Columbia Press.

Rodman Margaret et Jan Rensel, 1997. Home in the Islands: housing and social change in the Pacific, Honolulu, University of Hawai'i Press.

Rutz Henry, 1984. Material affluence and social time in village Fiji, in R.F. Salisbury and E. Tooker (eds), Affluence and cultural survival, Proceedings of the American ethnological society 1981, pp. 105-118.

SAmson Jane, 2009. Christianity, masculinity and authority in the life of George Sarawia, Journal of the Canadian Historical Association 20, pp. 60-84.

SCARr Derek, 1967. Fragments of Empire, a history of the Western Pacific High Commission, 1877-1914, Canberra, ANU Press.

Shineberg Dorothy, 1967. They came for Sandalwood, Melbourne, Melbourne University Press.

- 1999. The People Trade, Pacific Island Laborers and New Caledonia, 1865-1930, Honolulu, University of Hawai'i Press.
SPeISER Felix, 1996 [1923]. Ethnology of Vanuatu: an early twentieth century study, Honolulu, University of Hawai'i Press.

Stasch Rupert, 2011. Korowai treehouses and the everyday representation of time, belonging and death, The Asia Pacific Journal of Anthropology 12, pp. 327-347.

TaBAni Marc, 2002. Les pouvoirs de la coutume à Vanuatu: traditionalisme et édification nationale, Paris, L'Harmattan.

TAYLOR John, 2008. The other side: ways of being and place in Vanuatu, Honolulu, University of Hawai'i, Center for Pacific Studies, University of Hawai'i Press.

Thomas Nicholas, 1990. Sanitation and seeing. The creation of state power in early colonial Fiji, Comparative Studies in Society and History 32, pp. 149-170.

VeA Isileli, 1985. Changing shape of traditional house forms in Tonga: A case study, Lae, Architecture \& Building Dept., Papua New Guinea University of Technology.

VIenne Bernard, 1971. L'évolution de l'organisation politique des Motlaves (Nouvelles- Hébrides), Nouméa, оRSTOM

-, 1972. Les formes du pouvoir et de l'autorité dans les communautés paysannes des îles Banks, Nouméa, ORSTOM

—, 1982. Les masques Tamate des îles Banks (Vanuatu), Nouméa, ORSTOM

—, 1984. Gens de Motlav : Idéologie et pratiques sociales en Mélanésie, Paris, Société des Océanistes, Publications de la SdO 42.

—, 1996. Visages masqués du pays des morts, in C. Kaufmann et al. (éds), Vanuatu Océanie, arts des îles de cendre et de corail, Paris, RMN, ORSTOM, pp. 240-253.

Waterson, Roxana, 1990. The Living House: An Anthropology of Architecture in South-East Asia, Oxford, Oxford University Press.

Widmer Alexandra, 2008. The Effects of Elusive Knowledge: Census, Health Laws and Inconsistently Modern Subjects in Early Colonial Vanuatu, Journal of Legal Anthropology 1, pp. 92-116.

Wilson Cecil, 1895. Old scenes viewed through new glasses, Southern Cross Log 4, pp. 2-10.

-, 1911. Endeavour to Purify the Church in the Banks Islands, Southern Cross Log 16, pp. 147-154. 
